\title{
Daños de salud respiratoria en comunas expuestas a centrales termoeléctricas a carbón en el norte de Chile: análisis de datos secundarios
}

\author{
PAOLA VIVIANI G.***, CINTHYA LEIVA C.***, MARÍA JOSÉ OJEDA V.***, \\ ERICKA AHUMADA P.**** y SANDRA CORTÉS A.*,**,***
}

\section{Respiratory health damage in communes exposed to coal-fired power plants in northern Chile}

Introduction: In Chile, coal-fired power plants (CTEC) operate represents public health problems due to the health consequences for the population. Damage to the respiratory system is reported. Materials and Methods: Ecological study to evaluate hospital discharges in people living in cities where CTEC operates (Tocopilla and Huasco) were compared to a control city without CTEC (Caldera), all of them with similar sociodemographic conditions. Morbidity rates and Standardized Morbidity Ratios (SMR) were calculated. Results: The respiratory disease morbidity rate in Tocopilla (2016) was 152.5 per 10,000 inhabitants (habs) and for Huasco it is 135.2 per 10,000 inhabitants. In the control city, Caldera, the rate is 40.9 per 10,000 habs. In addition, the inhabitants of Tocopilla have a 2.42 times increased risk of bronchitis or bronchiolitis, 90\% higher risk of chronic lower respiratory diseases and 2.14 times more risk of asthma sickness. In Huasco, the population is 2.49 times more at risk of lower respiratory diseases compared to the region and 3.19 times more at risk of asthma. Conclusions: Asthma and bronchitis are the pathologies that showed the greatest risks in the cities of Tocopilla and Huasco. Our findings are similar to other research conducted in communities exposed to coal-fired power plants. Urgent action is needed to protect the health of the population.

Key words: Coal-fired power plants; Respiratory diseases; Chile; environmental pollution.

\section{Resumen}

Introducción: En Chile, el funcionamiento de las Centrales Termoeléctricas de Carbón (CTEC) representan un problema de salud pública debido a las consecuencias sanitarias que tienen en la población. Se han notificado daños en el sistema respiratorio. Materiales y Métodos: Se realizó un estudio ecológico, para evaluar los egresos hospitalarios de las personas que viven en las ciudades donde operan las CTEC (Tocopilla y Huasco), se comparó con una ciudad de control sin CTEC (Caldera), las comunas evaluadas tienen condiciones sociodemográficas similares. Se calcularon las tasas de morbilidad y tasas de morbilidad estandarizadas (SMR). Resultados: La tasa de morbilidad por enfermedades respiratorias en Tocopilla (2016) fue de 152,5 por 10.000 habitantes (habs) y para Huasco es de 135,2 por 10.000 habs. En la ciudad control, Caldera, la tasa es de 40,9 por 10.000 habs. Además, los habitantes de Tocopilla tienen 2,42 más riesgo de padecer bronquitis o bronquiolitis, 90\% más riesgo de presentar enfermedades crónicas de las vías respiratorias bajas y 2,14 veces más riesgo de enfermar por asma. En Huasco, la población tiene 2,49 veces más riesgo de padecer enfermedades de las vías respiratorias inferiores con respecto a la región y 3,19 veces más riesgo de presentar asma. Conclusiones: El asma y la bronquitis, son las patologías que mostraron mayores riesgos en las ciudades de Tocopilla y Huasco. Nuestros hallazgos son similares a otras investigaciones realizadas en comunidades expuestas a centrales termoeléctricas de carbón. Es necesario tomar medidas urgentes para proteger la salud de la población.

Palabras clave: Termoeléctricas a carbón; enfermedades respiratorias; Chile; contaminación ambiental.

\footnotetext{
* Advanced Center for Chronic Diseases, ACCDIS.

** Centro de Desarrollo Urbano Sustentable, CEDEUS.

*** Departamento de Salud Pública, Facultad de Medicina, Pontificia Universidad Católica de Chile. Santiago, Chile.

**** Comité de Salud Ambiental Infantil, Sociedad Chilena de Pediatría.
} 


\section{Introducción}

El desarrollo económico en Chile, está en parte asociado al hecho que el país es escenario de grandes instalaciones industriales a lo largo del territorio. Estas instalaciones industriales son conocidas por emitir altas cantidades de sustancias potencialmente tóxicas, tanto en el entorno laboral, como en el entorno general. Entre las sustancias que se emiten al ambiente, gran parte de ellas se transmiten a través de diversas vías generando impacto en la salud de la población ${ }^{1}$.

Las centrales termoeléctricas son parte de las grandes industrias que operan en el territorio nacional, muchas de ellas están basadas en el uso del carbón, lo que representa un potencial riesgo para la salud de las comunidades expuestas. Según Cortés et al. ${ }^{2}$, durante el ciclo de vida que tiene el carbón desde la extracción hasta los desechos de su postcombustión, se generan diversas emisiones, de partículas y gases, todas asociadas a daños en la salud de las personas.

En Chile, existe escasa evidencia de los daños en salud poblacional, aun cuando se presentan 27 unidades de generación eléctrica a carbón, ubicadas concentradamente en 5 ciudades a lo largo del país. En el año 2016, Ruiz et al. publicaron un estudio nacional que estableció el impacto de varias mega fuentes emisoras ubicadas en distintas comunas de Chile; se estimó un aumento de entre $20 \%$ y $100 \%$ en mortalidad y morbilidad por varias causas, entre ellas, causas cardiovasculares, respiratorias y cáncer al comparar con otras comunas sin megafuentes emisoras ${ }^{1}$.

El objetivo de este estudio fue describir la morbilidad en la zona norte de Chile en el período 2006-2016 para comunas expuestas de manera exclusiva a centrales termoeléctricas a carbón (CTEC) y compararlas con una comuna control. Lo anterior consideró describir las tasas de morbilidad general y de morbilidad específica por causas respiratorias por grupos de edad. Se consideró además estimar el riesgo de enfermar de la población de cada comuna respecto a su región.

\section{Caracterización del área de estudio}

En el norte de Chile existen CTEC en zonas cercanas a la población. Tocopilla y Huasco, son comunas localizadas en la región de Antofagasta y Atacama, respectivamente, donde operan CTEC (Figura 1). Ambas comunas, desde el año 2010 en Tocopilla y 2017 en Huasco, cuentan con un Plan de Descontaminación que tiene como objetivo mejorar las condiciones de calidad de aire según las normas establecidas. Estos planes han sido elaborados debido a que las condiciones de calidad de aire no son aceptables para la salud de las personas. Dentro de los contaminantes que emiten las CTEC se encuentran el material particulado (MP), dióxido de nitrógeno $\left(\mathrm{NO}_{2}\right)$, dióxido de azufre $\left(\mathrm{SO}_{2}\right)$, dióxido de carbono $\left(\mathrm{CO}_{2}\right)$, óxido de nitrógeno (NO) y metales pesados como el mercurio $(\mathrm{Hg})^{3}$.

Dichos contaminantes generan efectos en la salud de las personas, dentro de ellos, daños asociados a la salud respiratoria. Por ello, se estudió la morbilidad asociada a patologías del sistema respiratorio en comunas expuestas a CTEC. Además, se realizó el mismo análisis para Caldera, comuna localizada en la región de Atacama, que fue elegida como control por no tener termoeléctrica a carbón y estar localizada en zona costera como es el caso de las comunas expuestas.

Se listan en la Tabla 1, las principales características de las comunas seleccionadas para el período estudiado. Tocopilla fue la comuna con mayor concentración de MP y de $\mathrm{SO}_{2}$ mientras que Caldera es la que presentó las concentraciones más bajas. Tocopilla además es la que tuvo mayor cantidad de población con una densidad poblacional similar a la de Huasco. Por otro lado, Tocopilla y Caldera presentaron una mayor tasa de pobreza multidimensional que Huasco. Se observó además que las 3 comunas tienen un comportamiento similar en cuanto a la escolaridad del jefe de hogar y que en Caldera el porcentaje de la población que se declaró laboralmente activo fue mayor que en Tocopilla y Huasco.

\section{Materiales y Método}

\section{Tipo de estudio}

Se realizó un estudio epidemiológico de tipo ecológico. Estos estudios tienen la ventaja de caracterizar el estado de salud de una comunidad usando datos agregados, comparando tasas de ocurrencia de un evento en salud ${ }^{7}$ (Borja 2018).

\section{Indicadores de salud y análisis estadístico}

Los daños a evaluar fueron morbilidad general y por causas respiratorias. En la Tabla 2 se presentan las patologías seleccionadas junto al código de Clasificación Internacional de Enfermedades CIE10 ${ }^{8}$. Se utilizaron grupos de edades definidos en la literatura9. Para realizar los análisis, se consideraron las bases de egresos hospitalarios publicadas por el Departamento de Estadísticas e Información de Salud (DEIS) del Ministerio de Salud del período 2006 a 2016. Además, se utilizó información de las proyecciones de población realizadas por el Instituto $\mathrm{Na}$ - 


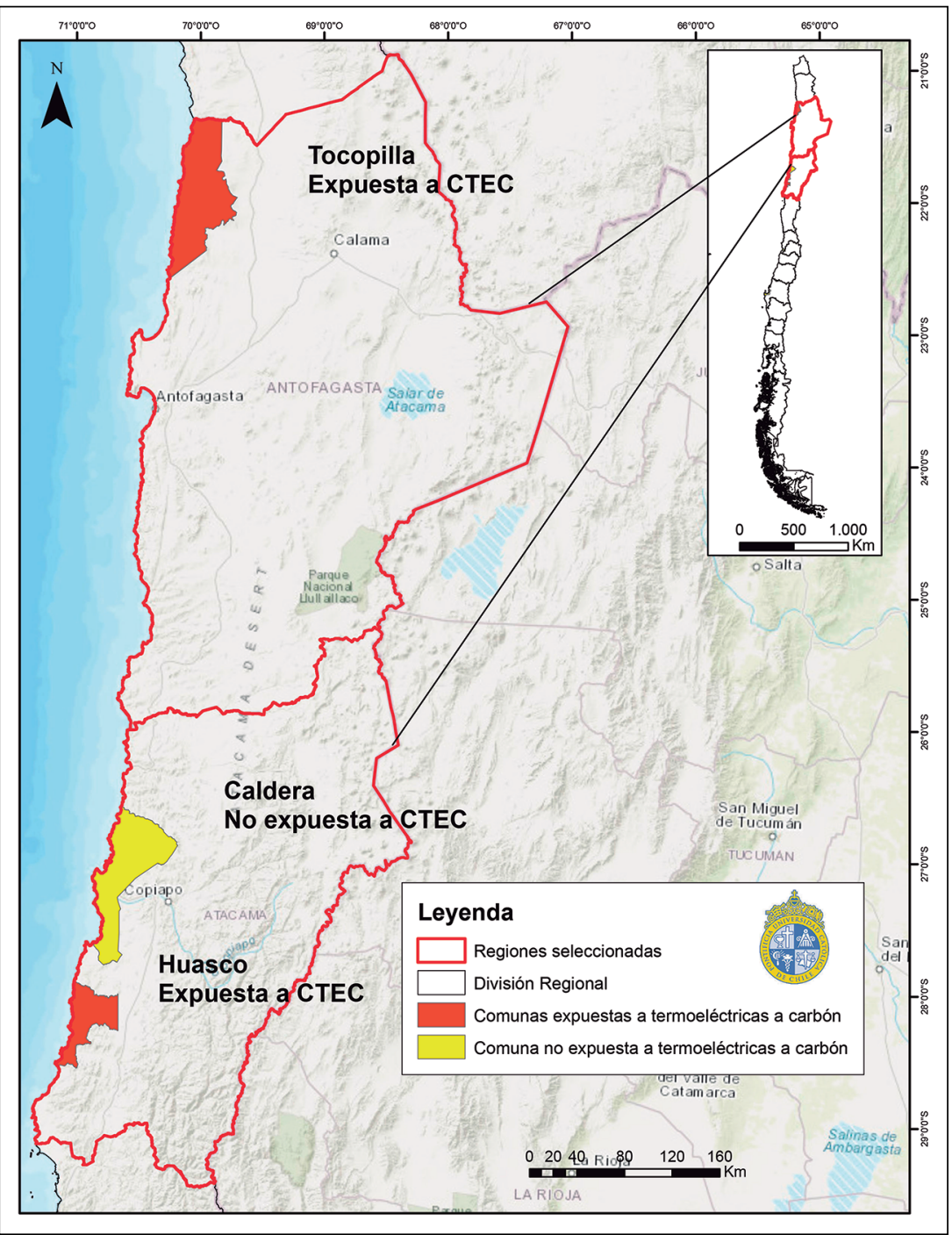

Figura 1. Mapa de localización del área de estudio.

Tabla 1. Características ambientales y sociodemográficas de Tocopilla, Huasco y Caldera

\begin{tabular}{|c|c|c|c|}
\hline Variables & Tocopilla & Huasco & Caldera \\
\hline Exposición CTEC & Exposición exclusiva & Exposición exclusiva & No expuesta \\
\hline $\mathrm{MP}(\mathrm{T} / \mathrm{año})^{\mathrm{a}}$ & $3.679,7$ & $1.851,5$ & 5,2 \\
\hline $\mathrm{SO}_{2}(\mathrm{~T} / \mathrm{año})^{\mathrm{a}}$ & 15.916 & $10.586,8$ & 6,1 \\
\hline Temperatura $\left({ }^{\circ} \mathrm{C}\right)^{\mathrm{b}}$ & $18,4(2015)$ & 15,2 & $\mathrm{~s} / \mathrm{d}$ \\
\hline Humedad del aire $(\%)^{b}$ & $63,9(2015)$ & 76,9 & $\mathrm{~s} / \mathrm{d}$ \\
\hline Velocidad del viento $(\mathrm{m} / \mathrm{s})^{\mathrm{b}}$ & 1,71 & 2,6 & $\mathrm{~s} / \mathrm{d}$ \\
\hline Población ${ }^{\mathrm{c}}$ & 25.186 & 10.149 & 17662 \\
\hline Densidad poblacional $\left(\mathrm{hab} / \mathrm{km}^{2}\right)^{\mathrm{c}}$ & 6,2 & 6,3 & 4,8 \\
\hline Tasa de pobreza multidimensional $(\%)^{\mathrm{d}}$ & 20,1 & 17,3 & 20,4 \\
\hline Escolaridad jefe/a hogar (media años) ${ }^{\mathrm{c}}$ & 10,5 & 10,2 & 10,6 \\
\hline Declaran trabajar $(\%)^{\mathrm{c}}$ & 53 & 58 & 61 \\
\hline
\end{tabular}

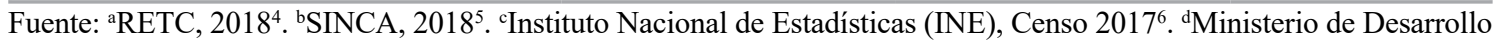
Social, CASEN, 2017. $\mathrm{T}=$ toneladas; hab = habitantes; $\mathrm{s} / \mathrm{d}=\sin$ datos. 
Tabla 2. Indicadores de morbilidad según clasificación CIE10 asociados a la exposición a contaminantes del aire

\begin{tabular}{|lcccc|}
\hline Indicador & Nombre común & $\begin{array}{c}\text { Código } \\
\text { CIE 10 }\end{array}$ & $\begin{array}{c}\text { Grupo } \\
\text { etario }\end{array}$ & Contaminante \\
\hline $\begin{array}{l}\text { Egresos hospitalarios Enfermedades } \\
\text { sistema respiratorio }\end{array}$ & $\begin{array}{c}\text { Egresos por enfermedades } \\
\text { respiratorias }\end{array}$ & $\mathrm{J} 00$ - J99 & $\begin{array}{c}\text { Todas las } \\
\text { edades }\end{array}$ & $\mathrm{MP}_{2,5}, \mathrm{O}_{3}$ \\
\hline $\begin{array}{l}\text { Egresos hospitalarios Enfermedades cró- } \\
\text { nicas de las vías respiratorias inferiores }\end{array}$ & $\begin{array}{c}\text { Egresos por enfermedades } \\
\text { del pulmón }\end{array}$ & $\mathrm{J} 40$ - J44 & $\begin{array}{c}\text { Todas las } \\
\text { edades }\end{array}$ & $\mathrm{MP}_{10}, \mathrm{NO}_{2}$ \\
\hline $\begin{array}{l}\text { Egresos hospitalarios Asma } \\
\text { Egresos por Asma }\end{array}$ & $\mathrm{J} 45-\mathrm{J} 46$ & $\begin{array}{c}\text { Todas las } \\
\text { edades }\end{array}$ & $\mathrm{MP}_{10}, \mathrm{NO}_{2}$ \\
\hline $\begin{array}{l}\text { Egresos hospitalarios por Bronquitis o } \\
\text { bronquiolitis aguda }\end{array}$ & Bronquitis aguda & $\mathrm{J} 20-\mathrm{J} 21$ & $\begin{array}{c}\text { Todas las } \\
\text { edades }\end{array}$ & $\mathrm{MP}_{10}, \mathrm{NO}_{2}$ \\
\hline
\end{tabular}

Fuente: Elaboración propia en base a Andersen et al, 2012, Heroux et al, 2015, Xie et al, 2016 ${ }^{10-12}$.

cional de Estadística ${ }^{6}$ a partir de los resultados del CENSO 2012, concordante con la metodología de cálculo de tasas realizada por el DEIS.

La morbilidad general y por causas respiratorias, se calculó para las comunas de Tocopilla y Huasco (expuestas a CTEC) y para Caldera como comuna control.

Se calcularon series temporales de tasas de morbilidad general y por causas específicas. Las tasas se obtuvieron para población general y según grupos de edad. A partir de las tasas de morbilidad general y específicas, se calcularon Razones de Morbilidad Estandarizadas (RME) para el año 2016. La estandarización (o ajuste) de tasas es un método epidemiológico que permite comparar, por ejemplo, por áreas de estudio (en este caso, comunas con diferente exposición a CTEC) ajustando por variables de confusión como la estructura etaria. Así, las RME proveen una estimación del riesgo relativo de la población en las comunas estudiadas con respecto al riesgo de la población de su región. Una RME superior a 1 indica que el riesgo de enfermar es superior en la población estudiada que en la población de referencia.

Para los análisis estadísticos se utilizó el software SPSS Statistics estableciendo un nivel de confianza del $95 \%$.

\section{Resultados}

Las tasas de morbilidad general, en el período 2006-2016, tuvieron diferencias entre las comunas expuestas a CTEC y la comuna control (Figura 2). Durante la mayor parte del período estudiado Tocopilla fue la comuna con mayor tasa de egresos hospitalarios. El año 2016 Tocopilla presentó una tasa de $1.181,7$ por 10.000 habitantes (habs.), tasa superior a la de Huasco (752,7 por 10.000 habs), a la de Caldera (500,3 por 10.000 habs.), a la tasa regional de Antofagasta $(899,9$ por 10.000 habs.) y a la de Chile (932,15 por 10.000 habs). Durante el transcurso del período, Tocopilla y Huasco mostraron un descenso en la morbilidad. La tasa de Tocopilla por cada 10.000 habs. pasó de 1.722,3 en el 2006 a 1.181,7 en el año 2016 mientras que en Huasco pasó de $1.361,1$ a 752,7 por 10.000 habs. Para el año 2016, la tasa de Huasco fue menor que las del país y región, con una disminución en los egresos hospitalarios desde el año 2014.

Caldera no presentó una variación significativa en el tiempo en la tasa de morbilidad general, y ésta se mantuvo siempre inferior a la de Tocopilla, Huasco, la región y el país.

Las tasas de morbilidad por causa respiratoria del período 2006-2016 de Tocopilla y Huasco fueron siempre superiores a las tasas de la comuna de Caldera (Figura 3).

Desde el año 2006 la tasa de morbilidad de Tocopilla desciende de 176,1 a 152,5 por 10.000 habs. en el año 2016. En ambos años las tasas de Tocopilla fueron superiores a la referencia de la región de Antofagasta de 117,7 (año 2006) y 82,58 (año 2016) por 10.000 habs. y a Chile, 104,67 (año 2006) y 85,93 (año 2016) por 10.000 habs.

La tasa de morbilidad de Huasco para el año 2006 fue de 151,4 y desciende a 135,2 por 10.000 habs. en el año 2016. En ambos períodos, las tasas de Huasco fueron superiores a la referencia de la región de Atacama correspondiente a 97,96 (año 2006) y 79,41 (año 2016) por 10.000 habs. y a Chile, 104,67 (año 2006) y 85,93 (año 2016) por 10.000 habs.

Por otro lado, las tasas de morbilidad por enfermedades respiratorias de la comuna de Cal- 


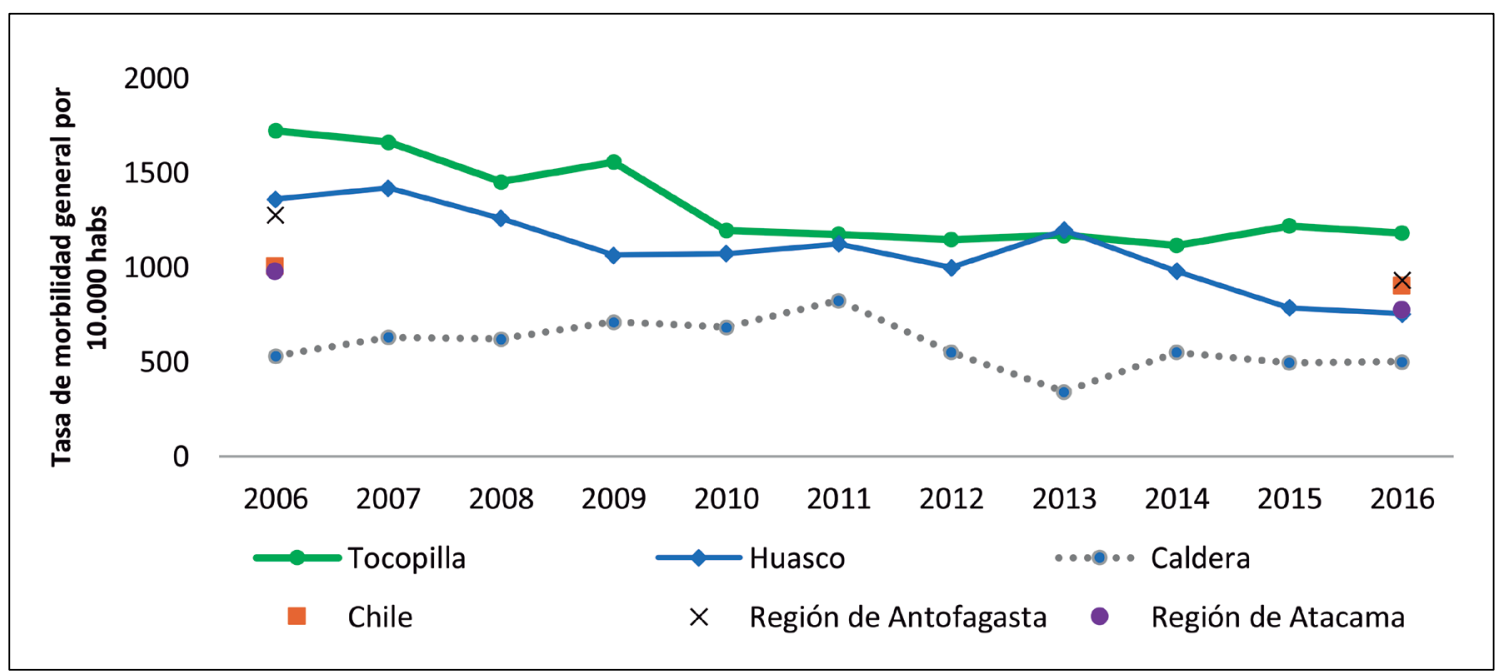

Figura 2. Morbilidad general en el período 2006-2016. $\beta$ Tocopilla $=-0,012$ Valor $p=0,001 . \beta$ Huasco $=-0,014$ Valor $\mathrm{p}=<0,001 . \beta$ Caldera $=-0,011$ Valor $\mathrm{p}=0,206$.

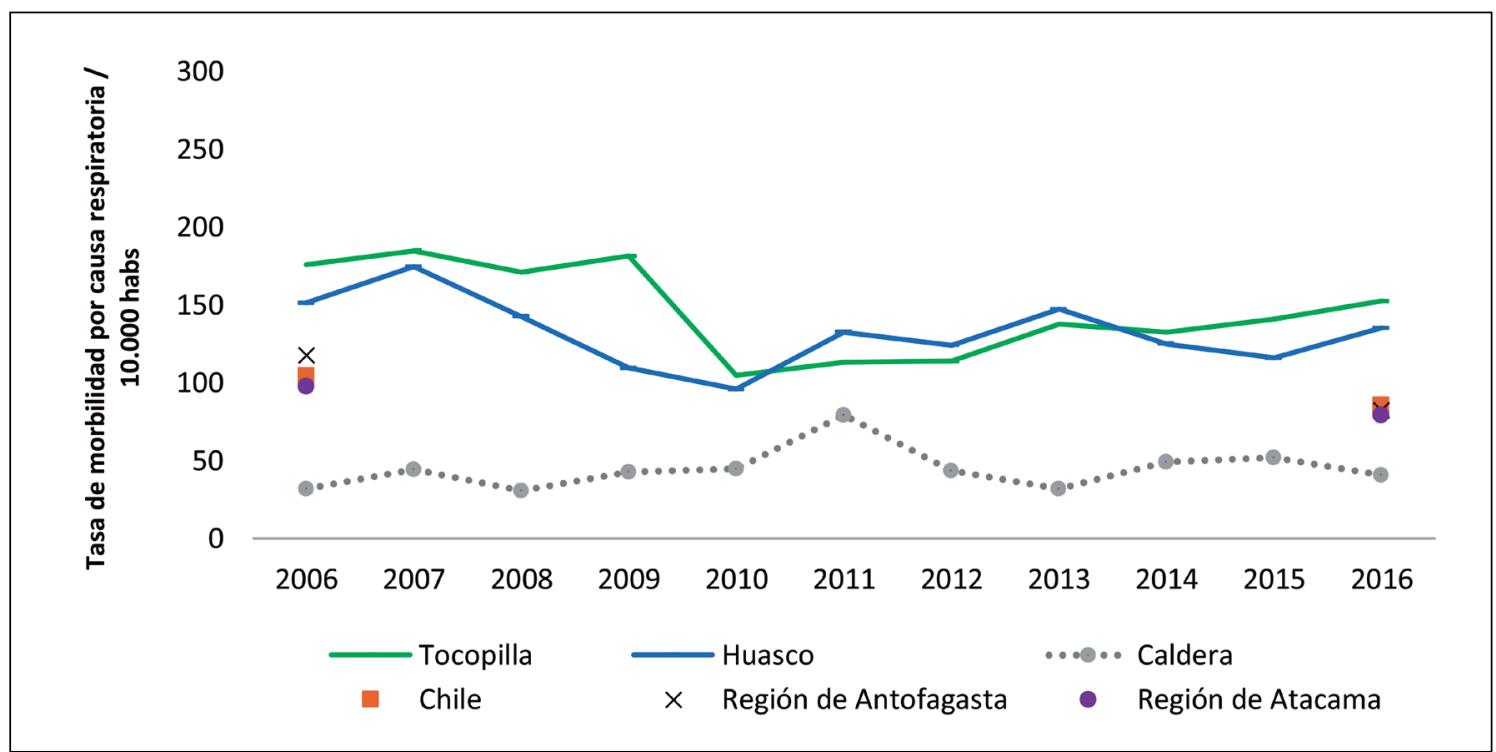

Figura 3. Morbilidad por causa respiratoria para los años 2006-2016.

dera corresponden a 31,9 (año 2006) y 40,9 (año 2016) por 10.000 habs.

En la Tabla 3 se observa que para el 2016 las regiones de Antofagasta y Atacama presentan tasas similares a las del país. Las dos comunas expuestas a CTEC tuvieron tasas de enfermedades respiratorias superiores a las tasas de Chile y la región de referencia en todos los grupos etarios excepto para el grupo de menores de 18 años en Huasco. Por otra parte, Caldera que corresponde a la comuna control, tuvo tasas inferiores respecto a Tocopilla, Huasco, al país y las regiones de Antofagasta y Atacama para todos los grupos etarios.

La comuna de Tocopilla presentó tasas por enfermedades del sistema respiratorio más elevadas que Huasco en todos los grupos etarios, a excepción del grupo de mayores de 80 años donde Huasco tiene una tasa de $1.809,05$ por 10.000 hab.

A partir de las RME (Tabla 4) se observa que los habitantes de Tocopilla presentaron un riesgo significativamente superior de enfermar en las tres patologías estudiadas respecto a su región. En particular, los habitantes de esta co- 
Tabla 3. Tasa de morbilidad específica por 10.000 habitantes por enfermedades respiratorias según territorio y grupos de edad, año 2016

\begin{tabular}{|ccccccc|}
\hline $\begin{array}{c}\text { Grupos de } \\
\text { edad (años) }\end{array}$ & Chile & $\begin{array}{c}\text { Región } \\
\text { Antofagasta }\end{array}$ & $\begin{array}{c}\text { Región } \\
\text { Atacama }\end{array}$ & Tocopilla & Huasco & Caldera \\
\hline $0-18$ & 144,97 & 141,38 & 129,77 & 190,58 & 122,33 & 79,64 \\
\hline $19-44$ & 27,79 & 30,87 & 23,69 & 47,13 & 38,82 & 11,67 \\
\hline $45-64$ & 44,58 & 54,22 & 41,43 & 111,57 & 100,00 & 19,65 \\
\hline $65-79$ & 164,53 & 169,73 & 177,36 & 364,39 & 290,76 & 71,32 \\
\hline$>80$ & 584,7 & 519,43 & 580,99 & 1185,03 & 1809,05 & 205,76 \\
\hline Total & 85,93 & 82,58 & 79,41 & 152,48 & 135,20 & 40,94 \\
\hline
\end{tabular}

Tabla 4. Razón de morbilidad estandarizada (respecto a la región) por bronquitis, enfermedades crónicas de las vías respiratorias inferiores y asma y en el año 2016

\begin{tabular}{|lcccccc|}
\hline Comuna & $\begin{array}{c}\text { Bronquitis o bronquiolitis } \\
\text { aguda }\end{array}$ & \multicolumn{2}{c|}{$\begin{array}{c}\text { Enfermedades crónicas de las } \\
\text { vías respiratorias inferiores }\end{array}$} & \multicolumn{2}{c|}{ Asma } \\
& CIE 10: J20-21 & \multicolumn{2}{c|}{ CIE 10: J40-44 } & \multicolumn{2}{c|}{ CIE 10: J45-46 } \\
RME & $\mathbf{9 5 \%}$ IC & RME & $\mathbf{9 5 \%}$ IC. & RME & $\mathbf{9 5 \%}$ IC \\
Caldera & 0,48 & $0,01-0,94$ & 0,43 & $0,05-0,80$ & 0,41 & $0,00-0,88$ \\
Tocopilla & 2,42 & $1,54-3,30$ & 1,90 & $1,33-2,47$ & 2,14 & $1,09-3,19$ \\
Huasco & 1,97 & $0,68-3,26$ & 2,49 & $1,40-3,58$ & 3,19 & $1,63-4,75$ \\
\hline
\end{tabular}

muna tuvieron 2,42 veces más riesgo de presentar bronquitis o bronquiolitis, $90 \%$ más riesgo de presentar enfermedades crónicas de las vías respiratorias inferiores y 2,14 veces más riesgo de enfermar por asma.

En la comuna de Huasco, la población tuvo 2,49 veces más riesgo de contraer enfermedades de vías respiratorias inferiores respecto a la región y 3,19 veces más riesgo de presentar asma. Los intervalos de confianza permiten concluir que estos resultados son estadísticamente significativos.

Adicionalmente, los habitantes de Caldera tuvieron RME significativamente inferiores a la región en las morbilidades específicas estudiadas, presentando en todos los casos valores inferiores a 1.

\section{Discusión}

El presente estudio describió y comparó el perfil de salud según tasas de morbilidad general y específica por causas respiratorias en comunas de la zona norte de Chile, con y sin CTEC.

A partir de los datos ambientales disponibles, se observó que de las 3 comunas estudiadas, las comunas expuestas tuvieron las mayores concentraciones de MP y de $\mathrm{SO}_{2}$, Se ha demostrado que las emisiones de MP debido a su tamaño, podrían permanecer suspendidas en el aire y depositarse en los pulmones al ser inhaladas, aumentando la morbilidad y mortalidad por enfermedades respiratorias, cardiovasculares, cerebrovasculares y por cáncer de pulmón ${ }^{13}$. Por otra parte, la exposición al $\mathrm{SO}_{2}$ emitido por las CTEC se ha asociado con una exacerbación de los síntomas respiratorios, y se ha correlacionado con tasas más altas de hospitalización por asma, especialmente entre niños y adultos mayores ${ }^{14}$. Sin embargo, la investigación sobre los efectos morfológicos o fisiológicos de estos contaminantes, continúa siendo limitada, y se ha basado principalmente en modelos animales. En estos estudios, se ha observado que las partículas de cenizas de carbón pueden causar alteraciones en el epitelio pulmonar y en células sanguíneas e inmunológicas, llevando a un mayor riesgo de enfermedades cardiopulmonares ${ }^{15-17}$.

Este estudio buscó identificar efectos en la salud de las personas en cuanto a su morbilidad medida según los egresos hospitalarios. Los resultados mostraron que las comunas expuestas a estas fuentes, en este caso Tocopilla y Huasco, 
tuvieron mayores tasas de morbilidad por enfermedades respiratorias respecto a Chile, a la región de referencia y a la comuna control.

Tomando en consideración los indicadores de riesgo los presentes resultados muestran que independiente sea el cuadro diagnóstico, residir en una comuna con CTEC conlleva un mayor riesgo de ser hospitalizado por causas respiratorias, que vivir en una comuna sin CTEC. El riesgo incluso llega a triplicarse para ser hospitalizado por asma. Por otro lado, se observó que el asma y bronquitis, fueron las patologías con mayor riesgo de hospitalización en las comunas de Tocopilla y Huasco. Los hallazgos coinciden con los de otras investigaciones de tipo ecológico, realizadas en comunidades expuestas a parques industriales o a CTEC, y que muestran mayor frecuencia de síntomas respiratorios, mayor número de consultas de urgencia por causas respiratorias, y mayor número de diagnósticos de asma y neumonías principalmente en niños que residen en zonas altamente expuestas ${ }^{2}$, lo cual se encuentra principalmente asociado a partículas y a hidrocarburos aromáticos policíclicos ${ }^{18}$.

El presente estudio mostró que para todas las unidades territoriales consideradas la tasa de morbilidad resultó más alta en niños y en adultos mayores. Con respecto a los niños se ha planteado que sus características físicas, su comportamiento y la inmadurez de su sistema respiratorio, entre otros factores, los hace más proclives a verse afectados por la contaminación del aire ${ }^{19}$. Para los adultos mayores en tanto, el deterioro de los tejidos y de su sistema inmune asociado a la edad, además de la presencia de otras enfermedades, podrían explicar su mayor vulnerabilidad a la exposición.

En la literatura internacional, los resultados coinciden con lo presentado en esta investigación. En Estados Unidos, en estudios poblacionales realizados en zonas cercanas a CTEC, se reporta que $85 \%$ de los padres informaron que sus hijos (niños) sufrían trastornos respiratorios ${ }^{20}$. Estudios realizados en China y Europa han arrojado que los contaminantes relacionados con las emisiones de las CTEC, se asocian con un mayor riesgo de enfermedades respiratorias y cardiovasculares, especialmente en mujeres embarazadas, niños, adultos con enfermedades respiratorias crónicas $\mathrm{y}$ adultos mayores ${ }^{21-23}$.

Tras el análisis de las tasas de morbilidad general y específicas y su variación en el transcurso del tiempo, se observó que tanto en Tocopilla como en Huasco, ambas tasas disminuyen, incluso Huasco al final del período mostró tasas de morbilidad inferiores a las de Chile y de su región. A pesar del valioso descenso, las cifras siguen siendo mayores a una comuna que no tiene CTEC, evidenciando la importancia de políticas públicas y normas ambientales, que protejan la calidad del aire en comunas expuestas a las emisiones de las distintas industrias existentes en el país. Respecto a lo anterior también hay que destacar que los niveles de contaminantes de MP y $\mathrm{SO}_{2}$ están por sobre la norma nacional ${ }^{24-26}$. Estas excedencias son conocidas por las autoridades y por la población en estudio. Por ello, es que en ambas comunas se han desarrollado Planes de Descontaminación Ambiental. Según la Organización Mundial de la Salud (OMS), en casos similares asociados a fuentes identificadas que generan contaminantes de riesgo para las personas, todos los esfuerzos para mejorar la calidad del entorno deben ponerse en marcha cuando las condiciones ambientales son deficientes y evidenciamos daños en salud, que es lo que vemos que ocurre en Tocopilla y Huasco.

La OMS señala dos estrategias complementarias, partiendo por un control riguroso de las emisiones de todas las fuentes que puedan liberar contaminantes de riesgo. Para ello, es necesario caracterizar adecuadamente qué contaminantes se liberan y en qué niveles, para luego reducir al máximo todas las emisiones asociadas con daños en salud. Según la naturaleza de estas emisiones, puede incluso realizarse el cierre de las fuentes. Por otra parte, la OMS establece que se debe asegurar una adecuada atención de salud en todos los niveles, de manera de mitigar los daños, especialmente en los grupos más vulnerables, como lo son los niños y las mujeres embarazadas, así como en las personas mayores que suelen presentar comorbilidades.

Por esta razón, es perentorio seguir evaluando a estas comunidades para evidenciar como se va mejorando la salud de las personas a medida que se realicen acciones que disminuyan efectivamente las emisiones de fuentes como las CTEC.

Si bien estos resultados se enmarcan dentro de lo esperado por los altos niveles de contaminantes detectados en la zona, deben ser tomados como una primera aproximación al estado de salud de las comunidades evaluadas establecido a partir de una metodología de investigación, que utiliza datos de salud recolectados de manera rutinaria por la autoridad de salud. No se debe olvidar que, al tratarse de un estudio de tipo ecológico, sólo se considera la variación temporal y la comparación geográfica, en relación a la condición de exposición o no exposición a CTEC, por lo que se recomienda tomar estos resultados con cautela, ya 
que no se enfoca en la causalidad multifactorial de los daños en salud medidos, ni su relación con otros factores relacionados con morbilidad en las comunas en estudio.

Finalmente, se debe considerar que los efectos en la salud de la población asociados a los contaminantes que puedan ser emitidos por CTEC son de tipo multifactorial. Es entonces necesario establecer una agenda de investigación integral, que pueda medir otros factores de riesgo, entre ellos los Determinantes Sociales de la Salud, y que permita medir el rol de cada una de las variables específicas para las alteraciones respiratorias identificadas como las más relevantes en la población.

\section{Agradecimientos}

Este estudio fue financiado por Chile Sustentable S.A. A su vez, agradecemos al equipo de Chile Sustentable conformado por Claudia Fuentes, Pamela Poo y Sara Larraín, quienes participaron en la formulación, revisión y organización de reuniones durante la realización del estudio.

\section{Bibliografía}

1.- RUIZ P, ARIAS N, PARDO S, MEYER M, MESÍAS $\mathrm{S}$, GALLEGUILLOS $\mathrm{C}$, et al. Impact of large industrial emission sources on mortality and morbidity in Chile: A small-areas study. Environment International 2016; 92-93: 130-8.

2.- CORTÉS S, YOHANNESSEN K, TELLERÍAS L, AHUMADA E. Exposición a contaminantes provenientes de termoeléctricas a carbón y salud infantil: ¿Cuál es la evidencia internacional y nacional? Revi Chil Pediatr 2019; 90 (1): 102-14.

3.- HEALTH AND ENVIRONMENT ALLIENCE (HEAL). Boosting health by improving air quality in the Balkans. Published Diciembre 2017 [revisado el 19 de enero de 2018]. Disponible en https://www. envhealth.org/IMG/pdf/13.12.2017_-_boosting_health_by_ improving_air_quality_in_the_balkans_ied_briefing. pdf.

4.- REGISTRO DE EMISIONES Y TRANSFERENCIAS DE CONTAMINANTES (RETC). Ministerio del Medio Ambiente. Datos RETC 2018. Recuperado el 2 de enero de: http://www.retc.cl/datos-retc/

5.- SISTEMA DE INFORMACIÓN NACIONAL DE CALIDAD DEL AIRE (SINCA). Información histórica 2018. Recuperado el 2 de enero de 2019: https:// sinca.mma.gob.cl/

6.- INSTITUTO NACIONAL DE ESTADÍSTICAS (INEB). Demográficas vitales 2017. Recuperado el 29 de enero de: https://www.ine.cl/estadisticas/demograficas-y-vitales.

7.- BORJA V. Estudios ecológicos. Salud Pública de México 2000; 42 (6), noviembre-diciembre.

8.- MINISTERIO DE SALUD (MINSAL). Aprueba norma general técnica que establece uso de la décima revisión de la clasificación estadística internacional de enfermedades y problemas relacionados con la salud para la codificación de las causas de muerte y de enfermedad. N¹2008. Disponible en: https://repositoriodeis. minsal.cl/deis/rem2008/100408/NormatecnicaCIE10. pdf.

9.- NACIONES UNIDAS. Directrices provisionales sobre clasificaciones internacionales uniformes de edades. Informes estadísticos 1983; serie M, $\mathrm{n}^{\circ} 74$. Recuperado el 22 de junio de: https://unstats.un.org/unsd/publication/ SeriesM/SeriesM_74s.pdf

10.- ANDERSEN Z, KRISTIANSEN L, ANDERSEN K, OLSEN T, HVIDBERG M, JENSEN S, et al. Stroke and long-term exposure to outdoor air pollution from nitrogen dioxide: a cohort study. Stroke 2012; 43: 3205.

11.- HEROUX ME, ANDERSON HR, ATKINSON R, BRUNEKREEF B, COHEN A, FORASTIERE F, et al. Quantifying the health impacts of ambient air pollutants: recommendations of a WHO/Europe project. Int J Public Health. 2015; 60 (5): 619-27.

12.- XIE W, LI G, ZHAO D, XIE X, WEI Z, WANG W, et al. "Relationship between fine particulate air pollution and ischaemic heart disease morbidity and mortality." Heart 2015; 101 (4): 257-63.

13.- LINAK WP, YOO J-I, WASSON SJ, ZHUB W, WENDT JOL, HUGGINS FE, et al. Ultrafine ash aerosols from coal combustion: Characterization and health effects. Proceedings of the Combustion Institute 2007; 31 (2):1929-37.

14.- US ENVIRONMENTAL PROTECTION AGENCY. Regulatory Impact Analysis for the Final Clean Air Interstate Rule. Washington, DC: Office of Air and Radiation Report; 2005. https://www.epa.gov/sites/ production/files/2015-09/documents/finaltech08.pdf

15.- COSTA DL, DREHER KL. Bioavailable transition metals in particulate matter mediate cardiopulmonary injury in healthy and compromised animal models. Environ Health Perspect. 1997; 105 (Suppl 5): 1053-60.

16.- PROCTOR SD, DREHER KL, KELLY SE, RUSSELL JC. Hypersensitivity of prediabetic JCR: LA-cp rats to fine airborne combustion particle-induced direct and noradrenergic-mediated vascular contraction. Toxicol Sci. 2006; 90 (2): 385-91.

17.- GOLDSMITH CA, HAMADA K, NING Y, QIN G, CATALANO P, KRISHNA MURTHY G, et al. Effects of environmental aerosols on airway hyperresponsiveness in a murine model of asthma. Inhal Toxicol. 1999; 11 (11): 981-98.

18.- AMSTER E, LEW-LEVY C. Impact of Coal-fired 
Power Plant Emissions on Children's Health: A Systematic Review of the Epidemiological Literature. Int. J. Environ. Res. Public Health. 2019; 16: 2008-16; doi:10.3390/ijerph16112008

19.- AMERICAN ACADEMY OF PEDIATRICS COUNCIL ON ENVIRONMENTAL HEALTH. Children's unique vulnerabilities to Environmental Hazards. Pediatric Environmental Health, 3rd Edition Elk Grove Village, IL: American Academy of Pediatrics. 2012; 13-23.

20.- ZIEROLD KM, SEARS CG. Community views about the health and exposure of children living near a coal ash storage site. J Community Health. 2015; 40 (2): 357-63.

21.- LIANG F, ZHANG G, TAN M, YAN C, LI X, LI $\mathrm{Y}$, et al. Lead in children's blood is mainly caused by coal-fired ash after phasing out of leaded gasoline in Shanghai. Environ Sci Technol. 2010; 44 (12): 4760-65.

22.- KARAVUŞ M, AKER A, CEBECI D, TAŞDEMIR M, BAYRAM N, ÇALI Ş. Respiratory complaints and spirometric parameters of the villagers living around the
Seyitomer coal-fired thermal power plant in Kütahya, Turkey. Ecotoxicol Environ Saf. 2002; 52 (3): 214-20.

23.- TANG D, LI T-Y, LIU JJ, ZHOU Z-J, YUAN T, CHEN Y-H, et al. Effects of prenatal exposure to coalburning pollutants on children's development in China. Environ Health Perspect. 2008; 116 (5): 674-9.

24.- MINISTERIO DEL MEDIO AMBIENTE. Establece norma primaria de calidad ambiental para material particulado fino respirable MP10. 2001. Recuperado el 22 de junio de: https://sinca.mma.gob.cl/uploads/documen tos/73881f634e74a87884b626007d5e585f.pdf

25.- MINISTERIO DEL MEDIO AMBIENTE. Establece norma primaria de calidad ambiental para material particulado fino respirable SO2. 2010. Recuperado el 22 de junio https://www.leychile.cl/ Navegar?idNorma $=179878$

26.- MINISTERIO DEL MEDIO AMBIENTE. Establece norma primaria de calidad ambiental para material particulado fino respirable M'P 2,5. 2011. Recuperado el 22 de junio de: https://www.leychile.cl/ Navegar?idNorma $=1025202$.
Correspondencia a:

Dra. Sandra Cortés A.

Departamento de Salud Pública.

Pontificia Universidad Católica de Chile.

Diagonal Paraguay 362, Piso 2, Santiago, Chile.

E-mail: scortesn@uc.cl 